DESY 99-137

\title{
Global anomalies in Chiral Lattice Gauge Theory*
}

\author{
Oliver Bär and Isabel Campos \\ Deutsches Elektronen-Synchrotron, Notkestrasse 85, 22603 Hamburg (Germany)
}

As first realized by Witten an $S U(2)$ gauge theory coupled to a single Weyl fermion suffers from a global anomaly. This problem is addressed here in the context of the recent developments on chiral gauge theories on the lattice. We find Witten's anomaly manifests in the impossibility of defining globally a fermion measure that reproduces the proper continuum limit. Moreover, following Witten's original argument, we check numerically the crossing of the lowest eigenvalues of Neuberger's operator along a path connecting two gauge fields that differ by a topologically non-trivial gauge transformation.

\section{Introduction}

In [1] Witten pointed out the mathematical inconsistency of an $S U(2)$ gauge theory coupled to only an odd number of Weyl fermions.

The inconsistency arises when one considers the behaviour of the effective action under topologically non-trivial gauge transformations $\left(\pi_{4}[S U(2)]=\mathbb{Z}_{2}\right)$, i.e. those that cannot be continuously deformed to the identity mapping.

Let $g$ be one of those non-trivial mappings. The gauge fields $A_{\mu}$ and its gauge transformed

$A_{\mu}^{\mathrm{g}}=\mathrm{g} A_{\mu} \mathrm{g}^{-1}+\mathrm{g} \partial_{\mu} \mathrm{g}^{-1}$,

are not connected by some smooth gauge transformations. However, they are connected in the space of all gauge fields since it is a vector space. Thus

$A_{\mu}^{t}=(1-t) A_{\mu}+t A_{\mu}^{\mathrm{g}}, \quad t \in[0,1]$,

is a well defined potential which interpolates between $A_{\mu}$ and $A_{\mu}^{\mathrm{g}}$. Witten showed that along this trajectory an odd number of eigenvalues of the square root of the Dirac operator crosses zero, leading to a switch of sign in the fermionic determinant [1]. The theory is thus ill-defined because the fermion determinant cannot be defined in a gauge invariant and smooth way.

Afterwards, the Witten anomaly has been established by other arguments. In particular, the change in the phase of the effective action under

\footnotetext{
*based on the talks given by O. Bär and I. Campos. Presented at the International Symposium on Lattice Field Theory, June 29 - July 3, 1999, Pisa, Italy.
}

$S U(2)$ gauge transformations can be calculated using the so-called embedding technique [2]. Here one uses the fact that $S U(2)$ can be embedded in another group with a trivial $\pi_{4}$, say $S U(3)$. The gauge fields $A_{\mu}$ and $A_{\mu}^{g}$ are then connected by some smooth family $\Omega$ of gauge transformations in $S U(3)$. In this context the change in the phase of the effective action is given by [3]

$\Delta \Gamma_{\mathrm{eff}}(\Omega d \Omega)=i \frac{-i}{240 \pi^{2}} \int \operatorname{tr}\left[(\Omega d \Omega)^{5}\right]=i \pi$,

leading to the switch of sign in the fermionic determinant.

During the last years encouraging progress has been made to formulate chiral gauge theories on the lattice as has been summarised in [4]. Any proper lattice formulation of an $S U(2)$ theory coupled to one doublet of chiral fermions should exhibit the Witten anomaly. This has been studied in the context of the overlap formalism [5]. Our purpose is showing how global anomalies arise in the recently developed action formalism [6,7].

\section{Weyl fermions on the lattice}

Exact chiral symmetry [8] can be achieved on the lattice provided the Wilson-Dirac operator $D$ fulfils the Ginsparg-Wilson relation [9].

Having exact chiral symmetry allows us to split the fermion fields in left- and right-handed, independently transforming components [8, 10,11]. In particular, we can restrict ourselves to the left- 
handed fields imposing the constraints

$\hat{P}_{-} \psi=\psi$,

$\bar{\psi} P_{+}=\bar{\psi}$.

One of the new features of the present approach is that $\hat{P}_{-}=\frac{1}{2}\left(1-\gamma_{5}(1-a D)\right)$ depends on the gauge field. Let us discuss some of its geometrical implications.

Consider a path in configuration space $U_{\mathrm{t}}(x, \mu)$, where $t \in[0,1]$ is the path parameter. We define an unitary operator $Q_{\mathrm{t}}$ through the differential equation

$\partial_{t} Q_{t}=\left[\partial_{t} P_{t}, P_{t}\right] Q_{t}, \quad Q_{0}=\mathbb{1}$,

where $\left.P_{\mathrm{t}} \equiv \hat{P}\right|_{U=U_{\mathrm{t}}}$. The operator $Q_{\mathrm{t}}$ is such that $P_{\mathrm{t}} Q_{\mathrm{t}}=Q_{\mathrm{t}} P_{0}$. In this way $Q_{\mathrm{t}}$ is the transporter of $P_{\mathrm{t}}$ along the path.

One key point here is that if the path is a closed loop, the operator $Q_{1}$ is not necessarily the identity map. Indeed, $Q_{1} \neq \mathbb{1}$ is an indication of a non-trivial bundle structure of the gauge field. As a measure for this let us define the quantity

$\mathcal{T}=\operatorname{det}\left[1-P_{0}+P_{0} Q_{1}\right]$,

for all closed loops. Using the unitarity of $Q_{\mathrm{t}}$ one easily proves that $\mathcal{T}$ is a phase. In particular, for gauge fields in $S U(2)$, using charge conjugation symmetry and the reality properties of the $S U(2)$ representations we have $\mathcal{T}= \pm 1$.

Finally we remark that the composition law

$\mathcal{T}_{\left[\Gamma_{1} \circ \Gamma 2\right]}=\mathcal{T}_{\Gamma 1} \mathcal{T}_{\Gamma_{2}}$

holds for two closed loops that have the same starting point.

\section{Fermionic Measures}

The action of the classical gauge theory coupled to a single Weyl fermion reads

$S_{F, L}=a^{4} \sum_{x} \bar{\psi}(x)\left[P_{+} D \hat{P}_{-} \psi\right](x)$.

In order to set up the quantum theory we have to define a measure for the fields in the path integral. Here the difficulty arises due to the gauge field dependence of the constraint (4). In fact, an infinitesimal deformation $\eta_{\mu}(x)$ of the gauge field $U(x, \mu)$, induces a change in the phase of the fermionic measure given by the so-called measure term [0]

$\mathfrak{L}_{\eta}=i \sum_{j}\left(v_{\mathrm{j}}, \delta_{\eta} v_{\mathrm{j}}\right) \equiv a^{4} \sum_{x} \eta_{\mu}^{c}(x) j_{\mu}^{c}(x)$,

where $c$ is the colour index and $\left\{v_{\mathrm{j}}\right\}$ is a basis of left-handed fields at $U(x, \mu)$.

We are then left with a gauge dependent phase ambiguity which has to be fixed in order to achieve the gauge invariance and the locality of the effective action. In [6,7] the problem is solved by choosing a current $j_{\mu}(x)$, local and gauge invariant function of the gauge fields, and such that an integrability condition is fulfilled. This condition is formulated in terms of the Wilson line

$W=\exp \left\{i \int_{0}^{1} d t \mathfrak{L}_{\eta}\right\}$,

where $a \eta_{\mu}(x)=\partial_{t} U(x, \mu) U(x, \mu)^{-1} . W$ measures the total change of phase along a given path $U_{\mathrm{t}}(x, \mu)$ in the set of gauge fields.

The integrability condition states that for all closed loops in configuration space the Wilson line must satisfy

$W=\mathcal{T}$.

Let us discuss the meaning of this condition. A local and gauge invariant current defines $W$ only locally. In (12) it is pointed out that the current must take into account the global geometry of the bundle underlying the gauge field. Global anomalies arise when 12) is not satisfied.

In the classical continuum limit, requiring a local and gauge invariant effective action implies [7]

$j_{\mu}(x)=0+\mathcal{O}(a)$.

Therefore $W=1+\mathcal{O}(a)$ in this limit and the integrability condition can only be satisfied if $\mathcal{T}=1$ for all closed loops.

In the next section we will show that in the $S U(2)$ theory with a single Weyl fermion there are closed loops in configuration space on which $\mathcal{T}=-1$. Therefore the anomaly arises because the proper classical continuum limit cannot be reproduced. 


\section{SU(2) Global Anomaly}

Let $g$ be a non-trivial $S U(2)$ gauge transformation, and consider its lattice version acting on the classical vacuum, $U(x, \mu)=\mathbb{1}$.

There are three different paths in configuration space connecting the classical vacuum with its gauge transformed, $g(x) g(x+a \mu)^{-1}$ :

- $\Gamma_{1} \equiv\left[g(x)^{t} g(x+a \mu)^{-t}\right]$ pure gauge $S U(2)$

- $\Gamma_{2} \equiv\left[\Omega(t, x) \Omega(t, x+a \mu)^{-1}\right]$ pure gauge $S U(3)$

- $\Gamma_{3} \equiv\left(g(x) g(x+a \mu)^{-1}\right)^{t}$

$\Gamma_{1}$ has no continuum limit. $\Gamma_{2}$ is the lattice analogue of the $S U(3)$ pure gauge path defined in the introduction and $\Gamma_{3}$ is a lattice version of Witten's path (2).

Our aim is computing $\mathcal{T}$ on the closed loop $\left[\Gamma_{3} \circ-\Gamma_{1}\right]$. Using the composition law (8) we split $\mathcal{T}$ in the following way

$\mathcal{T}_{\left[\Gamma_{3} \circ-\Gamma_{1}\right]}=\mathcal{T}_{\left[\Gamma_{3} \circ-\Gamma_{2}\right]} \mathcal{T}_{\left[\Gamma_{2} \circ-\Gamma_{1}\right]}$.

Along pure gauge loops we have,

$\mathcal{T}=\exp \left\{-i \int_{0}^{1} d t \sum_{x} \omega_{t}^{a}(x) \mathcal{A}^{a}(x)\right\}$,

where $\mathcal{A}^{a}(x)$ is the anomaly on the lattice.

For the vacuum configuration the anomaly vanishes because it is invariant under translations but also odd under parity, $\mathcal{A}^{a}(-x)=-\mathcal{A}^{a}(x)$. That means $\mathcal{T}_{\left[\Gamma_{2} \circ-\Gamma_{1}\right]}=1$. This argument holds for the vacuum in $S U(N)$. However, in $S U(2)$ the anomaly is identically zero in any configuration.

We are then left with the calculation of $\mathcal{T}$ for the loop $\left[\Gamma_{3} \circ-\Gamma_{2}\right]$. To deal with it we convert the line integral into a surface integral. On this surface the vector potential depends on two parameters $(t, s)$. It can be shown [12] that

$\partial_{\mathrm{s}} \ln \mathcal{T}=\int_{0}^{1} d t \operatorname{Tr} \hat{P}_{-}\left[\partial_{s} \hat{P}_{-}, \partial_{t} \hat{P}_{-}\right]$.

The r.h.s. of equation (16) can be expanded in powers of the lattice spacing $a$ and to leading order one finds

$\partial_{\mathrm{s}} \ln \mathcal{T}=-i c_{2} \int_{0}^{1} d t \int d^{4} x d_{R}^{a b c} \epsilon_{\mu \nu \rho \sigma} \eta_{\mu}^{a} \xi_{\nu}^{b} F_{\rho \sigma}^{c}(17)$
Here we take over the notation of [7]. The deformations $\eta$ and $\xi$ corresponds to the $t$ and $s$ directions respectively. In (17) we substitute our parameterisation of the vector potential on the surface. Integrating over $s$ we finally end up with the integral (3) and find $\mathcal{T}_{\left[\Gamma_{3} \circ-\Gamma_{2}\right]}=-1$. Altogether we get $\mathcal{T}_{\left[\Gamma_{3} \circ-\Gamma_{1}\right]}=-1$.

We want to point out that our proof is not restricted to have the classical vacuum as starting configuration (although it would be sufficient for the theory to be inconsistent). Since $\mathcal{T}$ is a homotopy invariant, smooth deformations of the loop cannot change its value.

\section{Witten Anomaly}

Next we want to show that the global anomaly we discovered in the previous section can be brought into a form that its equivalence to Witten's anomaly [1] is transparent. It will turn out that if we restrict ourselves to a real fermion determinant, $\mathcal{T}=-1$ implies a change of sign along the loop $\left[\Gamma_{3} \circ-\Gamma_{1}\right]$.

To start with consider the function

$f(t)=\operatorname{det}\left(1-P_{+}+P_{+} D_{t} Q_{t} D_{0}^{\dagger}\right)$,

that is a smooth function of $t$ for smooth paths. In addition it is real if the gauge group is $S U(2)$. For closed loops that function satisfies

$f(0)>0, \quad f(1)=\mathcal{T} f(0)$,

if $D$ has no zero mode at the starting point. This implies that $f$ passes through zero an odd number of times for $0 \leq t \leq 1$ if and only if $\mathcal{T}=-1$.

Next one can show

$f^{2}(t)=\operatorname{det} D_{t} \operatorname{det} D_{0}^{\dagger}$.

If $D^{\dagger}=\gamma_{5} D \gamma_{5}$, the eigenvalues of $D$ come in complex conjugate pairs, i.e.

$\operatorname{det} D=\prod \lambda_{i} \lambda_{i}^{*}$.

According to (20) a passing through zero of $f(t)$ at $t_{0}$ implies a passing through zero of an odd number of eigenvalues $\lambda_{i}(t)$. One can prove this by expanding both $f$ and $\lambda_{i}$ around $t_{0}$. Hence we have found that for any given loop in field space, starting at a point where $D$ has no zero modes, 
an odd number of pairs of eigenvalues of $D$ cross zero if and only if $\mathcal{T}=-1$.

Hence we find the same behaviour of the eigenvalues that Witten proved in [1] using the AtiyahSinger index theorem and that he used to conclude a change of sign of the fermion determinant. We can find the same here. In general, the fermion determinant is given by [7]

$$
\operatorname{det} M_{t} \operatorname{det} M_{0}^{\dagger}=f(t) W^{-1} .
$$

If we define $j_{\mu} \equiv 1$ we end up with a real fermion determinant all along the path. According to the properties of $f$, we find that $\operatorname{det} M_{1} \operatorname{det} M_{0}^{\dagger}$ is negative. Therefore $\operatorname{det} M_{t}$ changes sign along a loop with $\mathcal{T}=-1$.

Note however, that a change of sign is closely connected to the definition of a real fermion determinant. If we allow for complex values too and define a current $j_{\mu}$ such that $W$ equals -1 at the end of the loop, the fermion determinant is single valued. In that case the anomaly shows up in the disguise of a conflict with the correct continuum limit of $j_{\mu}(13)$ and $W$.

\section{Spectral flow of Neuberger's operator}

In the last section we have seen that $\mathcal{T}=-1$ implies that an odd number of pairs of eigenvalues of $D$ cross zero. On a finite lattice it is possible to confirm this crossing by a numerical computation of the spectral flow of an appropriate lattice Dirac operator, as we will see now.

Neuberger's operator [13] is an example for a Dirac operator that satisfies the Ginsparg-Wilson relation. It is explicitly given by

$$
\begin{aligned}
a D & =1-A\left(A^{\dagger} A\right)^{-1 / 2}, \\
A & =1-a D_{w} .
\end{aligned}
$$

$D_{w}$ denotes the usual Wilson-Dirac operator. The eigenvalues $\lambda_{j}$ of $a D$ lie on a unit circle around 1 in the complex plane. They can be parameterised by an angle $\theta$ according to

$\lambda_{j}=1-e^{i \theta_{j}}$.

Furthermore the eigenvalues come in complex conjugate pairs $\lambda_{j}, \lambda_{j}^{*}$ due to $D^{\dagger}=\gamma_{5} D \gamma_{5}$.

We are interested in the spectral flow of Neuberger's operator along the loop $\Gamma=\left[\Gamma_{3} \circ-\Gamma_{1}\right]$.
According to the last section we expect an odd number of eigenvalues that cross zero and go over into its complex conjugate value.

Along $\Gamma_{1}$ the eigenvalues are constant as a function of $t$ because it is a path of gauge transformations. The non-trivial part is along $\Gamma_{3}$ where we numerically computed the eigenvalues. This has been done in two steps. First of all we computed the low lying eigenvalues of the hermitian operator $a D^{\dagger} a D$.

They are given by the squared magnitude $\left|\lambda_{j}\right|^{2}$ of the eigenvalues (25). In a second step we calculated the imaginary part of $\lambda_{0}$, i.e. the eigenvalue with the smallest imaginary part at the beginning of the path. This is sufficient for our purpose. Before discussing the results let us make some remarks concerning the numerical computation itself.

We used a power series expansion into Chebyshev polynomials for the inverse square root of $A^{\dagger} A$ in (23). The Conjugate Gradient algorithm [14, 15] has been employed for the computation of the eigenvalues. In that way both the truncation and the numerical error are theoretically well under control.

For numerical reasons the path we used differs slightly from $\Gamma_{3}$. We did not start at the classical vacuum configuration but at a constant gauge field instead. This results in a gap of the spectrum at $t=0$ and $t=1$ and is advantageous in the numerical computation.

Figure 1 shows the first six lowest lying eigenvalues of $a D^{\dagger} a D$ as a function of the path parameter $t$. The obvious symmetry of the spectrum is due to our path parameterisation and the symmetry properties of the particular $g(x)$ we used. The magnitude of all but one eigenvalue is unequal to zero for the whole path. Only the lowest eigenvalue becomes zero for $t=0.5$. Hence only the imaginary part of $\lambda_{0}$ may cross zero and changes sign. To see if it really changes sign we calculated directly the imaginary part of $\lambda_{0}$. The result is shown in fig. 2 and indeed we find a crossing. Hence only one pair of eigenvalues crosses zero and we numerically confirmed a spectral flow that we have expected.

The change of sign of the fermion determinant can now be shown very explicitly. In terms of the 


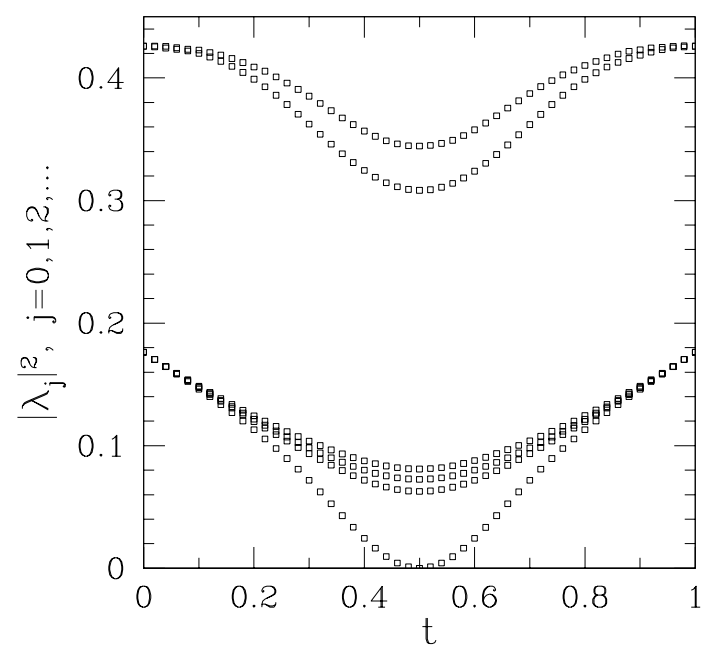

Figure 1. The lowest six eigenvalues of $a D^{\dagger} a D$ on a $8^{4}$ lattice. The total error is smaller than the size of the data points.

angles $\theta_{j}$ the fermion determinant reads as

$$
\operatorname{det} M_{t}=\prod_{j} 2 \sin \frac{\theta_{j}(t)}{2} \text {. }
$$

Here we fixed the phase of the fermion determinant such that det $M_{t}$ is real. However, we still have a sign ambiguity. Suppose we want to fix the sign but also insist on a smooth gauge field dependence of det $M_{t}$. This implies that the sign at $t=0$ fixes the sign along the whole path $\Gamma$. In terms of the angle $\theta_{j}$ our numerical result runs as follows: Only the angle $\theta_{0}$ crosses zero and changes sign. A glance at (26) immediately tells us that the fermion determinant changes sign along the closed path $\Gamma$ and is not a single valued function.

\section{Conclusions}

Let us give a brief summary of our result. We have shown that there are closed loops in configuration space on which the phase $\mathcal{T}=-1$. In a finite lattice it would be possible to define a current

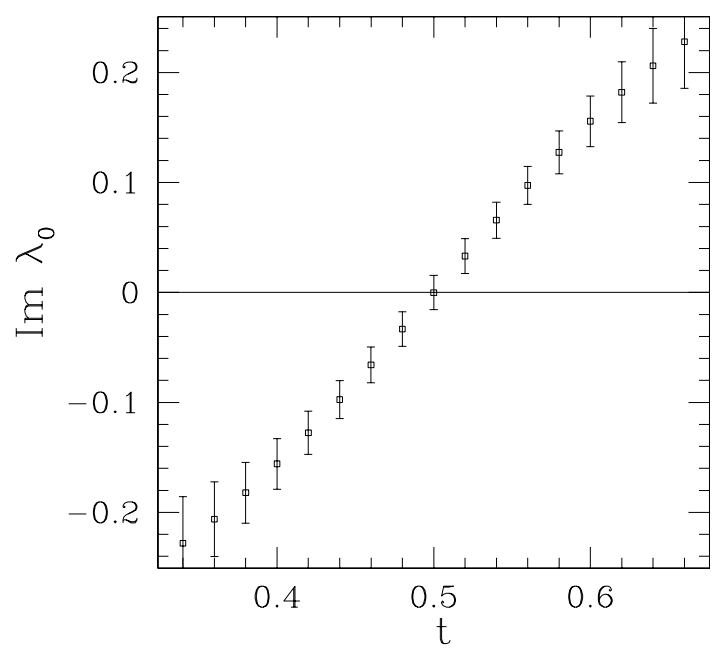

Figure 2. The imaginary part of $\lambda_{0}$. The error bars incorporate both truncation and numerical error.

$j_{\mu}(x)$ such that the associated measure produces a Wilson line $W=-1$. However, the conflict arises because the proper behaviour in the classical continuum limit cannot be reproduced: Going to large physical lattices (small lattice spacing) and insisting in $W=-1$, implies giving up the locality or the gauge invariance of the theory. Our argument is completely analytical, in particular the behaviour close to the continuum limit is under control.

We have also checked numerically the original argument given by Witten. We find that along a path connecting two gauge fields that differ by a topologically non-trivial gauge transformation, the lowest eigenvalue of Neuberger's operator crosses zero. Subsequently there exist closed loops in configuration space where the fermion determinant changes sign.

On the lattice all gauge transformations can be deformed to the identity mapping. As we have seen it does not mean that global anomalies are not present in the lattice formulation, but they rather arise in a different way. 


\section{Acknowledgements}

The authors are grateful to Martin Lüscher for helpful discussions and advise.

The numerical computations have been performed on the Linux cluster of the DESYHamburg Theory Group and on the RTNN Linux farm in Zaragoza (Spain).

\section{REFERENCES}

1. E. Witten, Phys. Lett. B117 (1982) 324.

2. S. Elitzur and V.P. Nair, Nucl. Phys. B243 (1984) 205.

3. E. Witten, Nucl. Phys. B223 (1983) 433.

4. M. Lüscher's plenary talk given at Lattice '99, Pisa (Italy).

5. H. Neuberger, Phys. Lett. B437 (1998) 117.

6. M. Lüscher, Nucl. Phys. B549 (1999) 295.

7. M. Lüscher, hep-lat:/9904009.

8. M. Lüscher, Phys. Lett. B428 (1998) 342.

9. P. H. Ginsparg and K. G. Wilson, Phys. Rev. D25 (1982) 2649.

10. F. Niedermayer, Nucl. Phys. B (Proc. Suppl.) 73 (1999) 105.

11. R. Narayanan, Phys. Rev. D58 (1998) 097501.

12. O. Bär and I. Campos, "Global Anomalies on the Lattice" (in preparation).

13. H. Neuberger, Phys. Lett. B417 (1998) 141 and Phys. Lett. B427 (1998) 453.

14. B. Bunk et. al., ALPHA collaboration internal report (unpublished) (1994).

15. T. Kalkreuther and H. Simma, Comp. Phys Comm. 93 (1996) 33. 